\title{
Parálisis del músculo trapecio por biopsia de ganglio cervical. Video ilustrativo
}

\author{
Trapezius muscle paralysis due to cervical ganglion biopsy. Illustrative video
}

\author{
Luis Gerardo Domínguez Carrillo*
}

Citar como: Domínguez CLG. Parálisis del músculo trapecio por biopsia de ganglio cervical. Video ilustrativo. Acta Med. 2021; 19 (1): 159-160. https://dx.doi.org/10.35366/98596

\begin{abstract}
Paciente femenino de 42 años que acude al servicio de rehabilitación buscando mejoría en la función de la extremidad superior izquierda. Como antecedente de importancia: le efectuaron biopsia de ganglio en cara anterolateral de cuello (zona II), con resultados histopatológicos de inflamación inespecífica, sin datos de malignidad; presentando de inmediato dolor moderado en zona quirúrgica con irradiación a nuca, hombro y brazo izquierdos, con dificultad inmediata para la movilización voluntaria del hombro al intentar abducción. A los 45 días de la toma de biopsia notó hundimiento de la región supraclavicular. A la exploración física se encontró descenso de hombro izquierdo, atrofia de músculo trapecio izquierdo y porción externa del esternocleidomastoideo izquierdo, hundimiento de fosa supraclavicular izquierda por atrofia del músculo trapecio, observándose trayecto del músculo omohioideo en el fondo de la fosa supraclavicular izquierda con hipotrofia de la porción externa del esternocleidomastoideo; cicatriz de $2 \mathrm{~cm}$ de longitud sobre borde anterior del músculo trapecio en la unión del tercio superior con tercio medio y perpendicular a éste, correspondiendo a la línea de división de las zona II y III del cuello; en la vista posterior se observó: desplazamiento lateral de la escápula y atrofia del trapecio; a la palpación: el trapecio superior da la sensación de cuerda fibrosa y la cicatriz mencionada está adherida a planos profundos; arcos de movimiento de hombro izquierdo activos, pero limitados como sigue: flexión $110^{\circ}$, abducción $45^{\circ}$, Video 1: Parálisis del músculo trapecio por biopsia de ganglio cervical.
\end{abstract}

\footnotetext{
* Especialista en Medicina de Rehabilitación, Catedrático de la Facultad de Medicina de León, Universidad de Guanajuato. México.
}

Correspondencia:

Acad. Dr. Luis Gerardo Domínguez Carrillo

Correo electrónico: Igdominguez@hotmail.com

Aceptado: 18-12-2019.

www.medigraphic.com/actamedica

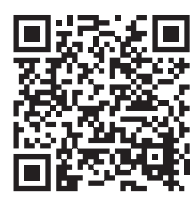

https://www.medigraphic.com/videos/actmed/am211ag rotación externa $50^{\circ}$, resto normales; movilidad pasiva completa; fuerza muscular valorada en escala de 0 a 5 en miembro superior izquierdo mostró: trapecio superior en 0 ; esternocleidomastoideo 4; romboides 4; angular del omóplato 4; deltoides 5; resto en 5 al igual que todos los músculos de miembro superior derecho, sensibilidad y reflejos normales. La funcionalidad del miembro superior izquierdo alcanzó 55 en la escala de DASH.

El nervio espinal es susceptible de lesiones, pues en su trayecto entre los músculos esternocleidomastoideo y trapecio se hace superficial encontrándose en el tejido subcutáneo en el triángulo posterior del cuello; la etiología de la lesión del nervio espinal, la cual ocasiona parálisis del músculo trapecio, es múltiple; la reportada con más frecuencia se presenta durante la biopsia o escisión de ganglios linfáticos ${ }^{1,2}$ o tumores benignos en el triángulo posterior del cuello.

Por ser tan largo tiempo de evolución se estableció programa de fortalecimiento de músculos deltoides, romboides, angular de la escápula y serrato mayor, derivando a la paciente a cirugía del miembro torácico para valorar las posibilidades de transposición muscular de la cabeza esternal del pectoral mayor a la escápula, descrita por Iceton y Harris en 1987. Tanto el grupo de Streit en 2012 como Elhassan y colaboradores en 2015 así como Li y su equipo en 2017 reportan excelentes y buenos resultados en la estabilización de la escápula, mejorando la función. ${ }^{3-6}$

\section{REFERENCIAS}

1. Park SH, Esquenazi Y, Kline DG, Kim DH. Surgical outcomes of 156 spinal accessory nerve injuries caused by lymph node biopsy procedures. J Neurosurg Spine. 2015; 23 (4): 518-525.

2. Ferraresi $S$, Basso E, Pino MA, Dipasquale P. Iatrogenic accessory nerve palsy after laterocervical lymph node biopsy-clinico-surgical considerations on 42 cases and review of the literature. Clin Surg. 
2017; 2: 1466-1470.

3. Iceton J, Harris WR. Treatment of winged scapula by pectoralis major transfer. J Bone Joint Surg Br. 1987; 69 (1): 108-110.

4. Streit JJ, Lenarz CJ, Shishani Y, McCrum C, Wanner JP, Nowinski RJ et al. Pectoralis major tendon transfer for the treatment of scapular winging due to long thoracic nerve palsy. J Shoulder Elbow Surg. 2012; 21 (5): 685-690.

5. Elhassan BT, Wagner RE. Outcome of transfer of the sternal head of the pectoralis major with its bone insertion to the scapula to manage scapular winging. J Shoulder Elbow Surg. 2015; 24 (5): 733-740.

6. Li T, Yang ZZ, Deng Y, Xiao M, Jiang C, Wang JW. Indirect transfer of the sternal head of the pectoralis major with autogenous semitendinosus augmentation to treat scapular winging secondary to long thoracic nerve palsy. J Shoulder Elbow Surg. 2017; 26 (11): 1970-1977. 